doi: http://dx.doi.org/10.7124/bc.0009EF

\section{M-2. The mechanisms of YB-1 nucleocytoplasmic translocation}

D. A. Mordovkina, I. A. Eliseeva, L. P. Ovchinnikov

Institute of Protein Reseach, Russian Academy of Sciences, Russian Federation

darja-mordovkina@rambler.ru

The Y-box binding protein 1 (YB-1) is a DNA and RNA binding protein that performs numerous functions both in the cytoplasm and in the nucleus. Its nuclear localization has been observed at the G1/S cell cycle boundary [1], in stress conditions of various types [2-5], and under adenovirus infection [6]. The sequence of YB-1 contains a nuclear localization signal (NLS) [7] which is responsible for its nuclear translocation. The YB-1 NLS is recognized by transportin 1 [8] and classified with signals of the PY-NLS type characterized by the presence of an N-terminal positively charged or hydrophobic cluster, the residue $\mathrm{R}$, and the $\mathrm{C}$-terminal dipeptide PY. For better understanding of NLS functioning, we generated a few forms of YB-1 with mutations in its NLS region, where major structural elements of NLS were modified. We compared subcellular localization of the WT YB-1 with that of its mutant forms both in normal growth conditions and with stimulation of its translocation to the nucleus. Methods: The mutations were performed by site-directed mutagenesis. Transfection of eukaryotic cells was made according to the manufacturer's recommendations. Results: The comparison of subcellular localization of WT YB-1 with that of its mutant forms showed that only the removal of the entire NLS resulted in the loss of the nuclear translocation ability of YB-1. The removal or replacement of separate structural elements of the NLS did not prevent YB-1 translocation to the nucleus either in normal growth conditions or under stimulated translocation. Conclusions: There is increasing evidence in the literature that some PY-NLS found in various proteins interact with transportin 1 . Besides, transportin 1 can recognize PY-NLS lacking the dipeptide PY [9-12]. The YB-1 NLS has a highly charged N-terminal cluster which presumably makes the greatest contribution to the interaction with transportin 1 .

This work was supported by the RFFI (\#18-34-00359 \18) References: 1. Jurchott, K., et al. (2003) J. Biol. Chem., 278, 27988-27996 2. Stein, U., et al. (2001) J. Biol. Chem., 276, 28562-28569 3. Das, S., et al. (2007) J. Biol. Chem., 282, 28474-28484. 4. Koike, K., et al. (1997) FEBS Lett., 417, 390-394. 5. Fujita, T., et al. (2005) Clin. Cancer Res., 11, 8837-8844. 6. Holm, P. S., et al. (2002) J. Biol. Chem., 277, 10427-10434. 7. Bader, A. G., et al. (2005) Mol. Cell. Biol., 25, 2095-2106. 8. Chook, Y.M., et al. (2011) Biochim. Biophys. Acta 1813, 1593-1606. 9. Soniat, M., et al. (2015) Biochem. J. 468, 353-362. 10. Twyffels, L., et al. (2014) FEBS Lett. 588, 1857-1868. 11. Soniat, M et al. (2016) Structure 24, 1802-1809.

doi: http://dx.doi.org/10.7124/bc.0009F0

\section{M-3. HIV 1 Tat induces cell type specific expression of host genes in $B$ cells}

M. Gorbacheva ${ }^{1}$, A. Valyaeva ${ }^{2}$, D. Potashnikova $^{3}$, E. Arifulin ${ }^{4}$, Y. Vassetzky ${ }^{1,5,6}$, Y. Musinova ${ }^{1,4}$

${ }^{1}$ Koltzov Institute of Developmental Biology, Russian Academy of Sciences, Moscow, Russia;

${ }^{2}$ Department of Bioengineering and Bioinformatics, 